\title{
Improved Virus Specimen Preparation for Transmission Electron Microscopy Using mPrep/g Capsules: Applications in BSL3-4 Laboratories
}

\author{
Mitchell K. Monninger ${ }^{1}$, Chrystal A. Nguessan ${ }^{1}$, Candace D. Blancett ${ }^{1}$, Steven L. Goodman ${ }^{2}$ and Mei G. \\ Sun $^{1}$ \\ 1. US Army Medical Research Institute of Infectious Diseases, Ft. Detrick, MD, USA \\ 2. Microscopy Innovations LLC, 213 Air Park Rd, Suite 101, Marshfield, WI, USA
}

Viruses are examined with TEM to screen for unknown disease causing agents, research structure, elucidate mechanisms, verify molecular biology assays, and make diagnoses [1-3]. Viruses are commonly prepared by applying viral suspension to filmed grids, followed by rinsing, negative staining with uranyl acetate, blotting, and storing in grid boxes until TEM imaging [4-5]. Each step requires careful use of fine forceps to move delicate grids between reagents and grid boxes. Such handling is much more difficult when preparing pathogenic viruses in a bio-containment Biosafety Laboratory (BSL3/4) since positive pressure suits with facemasks make it difficult to see grids and double layer gloves reduce dexterity. Finally, since TEMs are usually outside the BSL, grids must be treated with decontamination agents (fixatives), which add additional protocol steps.

We developed an easy and reproducible method to prepare pathogenic viruses in BSL4 biocontainment that eliminates TEM grid handling. Instead of handling grids with forceps, all preparation is done with grids safely encapsulated in mPrep/g capsules, thus removing sharp forceps as a puncture risk. Virus suspensions and other reagents are then delivered to grids using lab pipettors that are easily handled wearing protective gear. Many grids may be prepared simultaneously using multi-channel pipettors, even with different protocols or conditions. Reduced effort, consistent quality, and simultaneous preparation makes this method equally useful with non-pathogenic viruses. The method has been applied to large viruses such as Ebola filovirus (Figure 1a), small viruses, and virus like particles (Figure 1b).

Advance preparation: Formvar-filmed [4] 400 mesh $\mathrm{Cu}$ grids were inserted into mPrep/g capsules (Microscopy Innovations, LLC). The capsules were then transferred into the BSL4 biocontainment with mPrep/f 13 filter couplers, a pipettor, and 96-well plates. Also transferred into biocontainment were 2\% aqueous glutaraldehyde, and a container to deliver osmium tetroxide $\left(\mathrm{OsO}_{4}\right)$ vapor to $\mathrm{mPrep} / \mathrm{g}$ capsules.

BSL4 biocontainment: $\mathrm{mPrep} / \mathrm{g}$ capsules with filmed grids were connected to a pipettor using mPrep/f couplers that prevent reagents from entering the pipettor. Virus suspension was aspirated into the $\mathrm{mPrep} / \mathrm{g}$ capsules to cover the grids $(40 \mu \mathrm{l})$ and held for 10 minutes to allow the virus to settle and adsorb. The virus suspension was then dispensed. $2 \%$ glutaraldehyde was aspirated into the capsules for 10 minutes decontamination, and then grids were washed $3 \mathrm{x}$ with DI water. The $\mathrm{mPrep} / \mathrm{g}$ capsules were then removed from the pipettor and decontaminated by sealing in a container containing filter paper soaked with $1 \% \mathrm{OsO}_{4}$ for 1 hour. The container was then transferred to the EM lab.

Electron microscopy lab: mPrep/g capsules were removed from the $\mathrm{OsO}_{4}$ vapor container in a fume hood and attached to a pipettor using clean mPrep/f couplers. Grids were washed 3x with $40 \mu 1 \mathrm{DI}$ water and negatively stained with $1 \%$ uranyl acetate by holding the stain for 30 seconds, then dispensing. $\mathrm{mPrep} / \mathrm{g}$ capsules were removed and grids were wick-dried by inserting filter paper into the capsules and 
touching where the grid edges are held in the capsule. The grids were then removed for imaging at 80 $\mathrm{kV}$ with a JEOL 1011 TEM equipped with an AMT digital camera system [6].

References:

[1] A Curry, H Appleton, B Dowsett. Micron 37(2) (2006) p. 91.

[2] CS Goldsmith, SE Miller. Clinical Microbiology Reviews, October (2009) p. 552

[3] M Laue. Methods Cell Biol. 96 (2010) p. 1.

[4] JJ Bozzola, LD Russell, "Electron Microscopy” 2nd ed, (Jones and Bartlett, Boston) p.124.

[5] S Brenner, RW Horne. Biochim Biophys Acta 34 (1959) p.103.

[6] US Army Medical Research Institute of Infectious Disease (USAMRIID) Disclaimer: Opinions, interpretations, conclusions, and recommendations are those of the authors and are not necessarily endorsed by the U.S. Army.
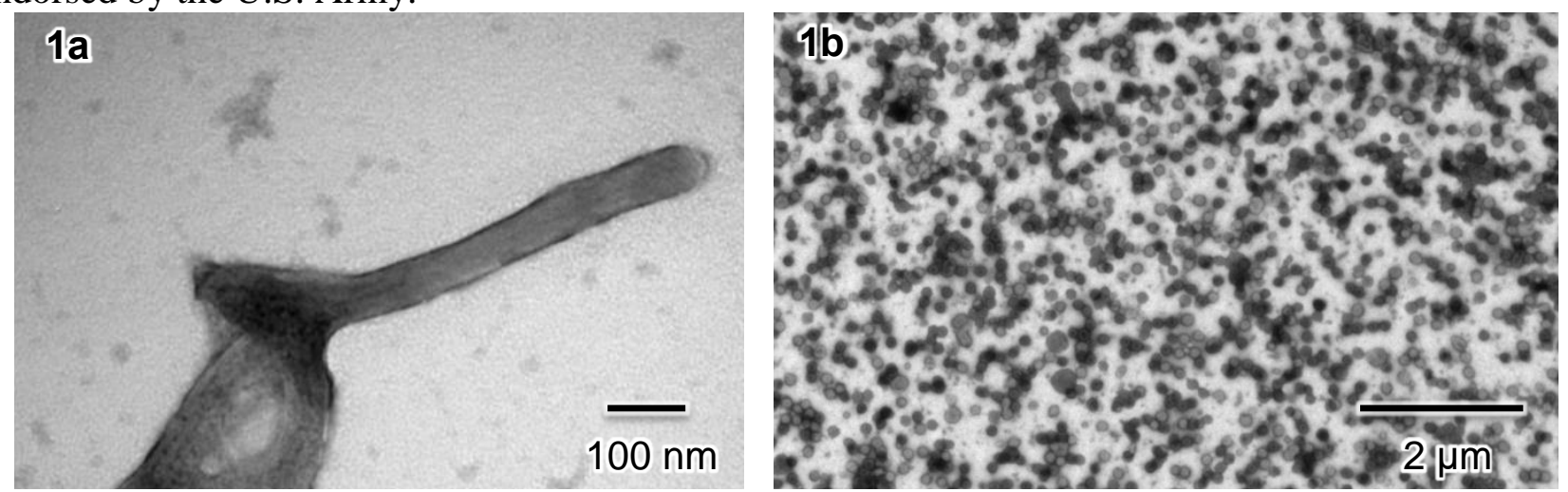

Figure 1: Ebola virus (a) and murine leukemia virus like particles (b).

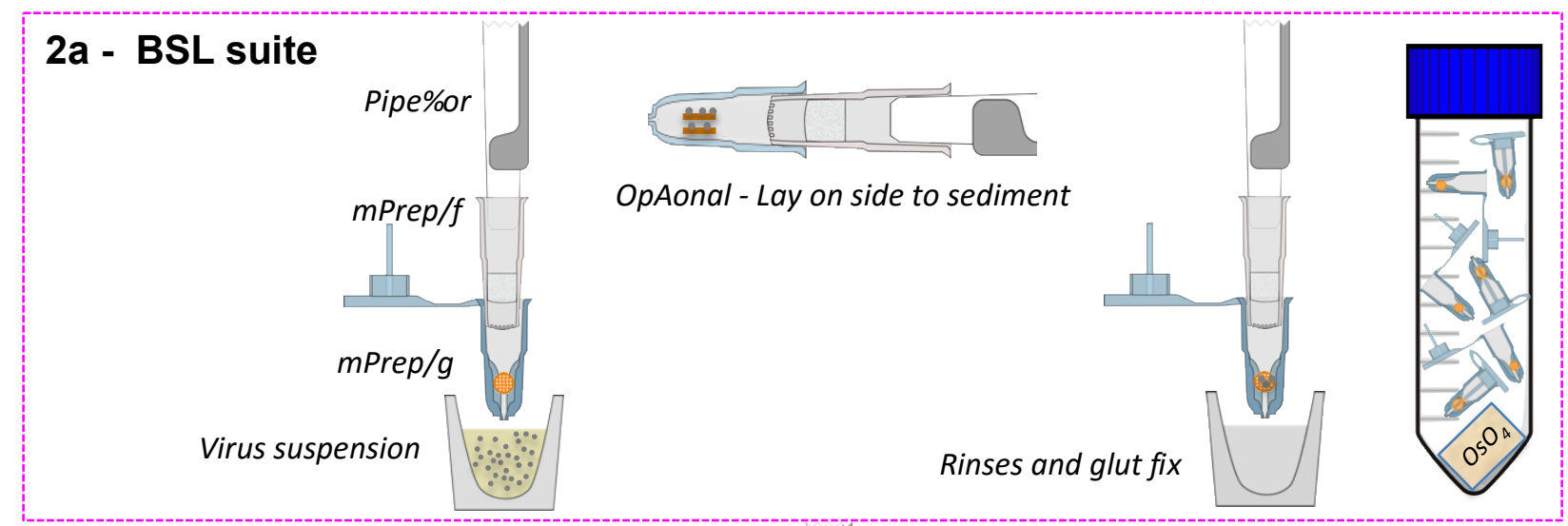

2b - EM lab

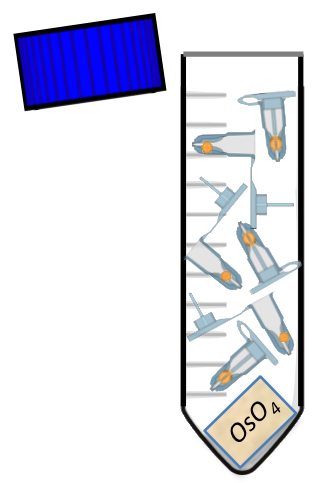

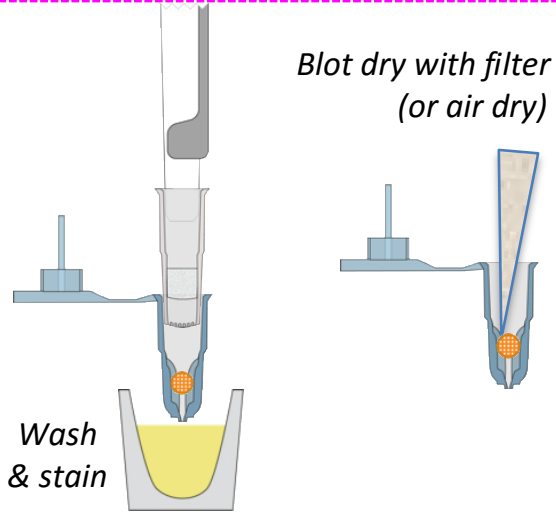

Figure 2: Diagram of protocol in BSL suite (a) and protocol in electron microscopy lab (b). 